\title{
Elemental Topological Insulator with Tunable Fermi Level: Strained $\alpha$-Sn on InSb(001)
}

\author{
A. Barfuss, ${ }^{1}$ L. Dudy,${ }^{1}$ M. R. Scholz,${ }^{1}$ H. Roth, ${ }^{1}$ P. Höpfner, ${ }^{1}$ C. Blumenstein, ${ }^{1}$ G. Landolt,${ }^{2,3}$ J. H. Dil, ${ }^{2,3}$ \\ N.C. Plumb, ${ }^{2}$ M. Radovic, ${ }^{2}$ A. Bostwick, ${ }^{4}$ E. Rotenberg, ${ }^{4}$ A. Fleszar, ${ }^{5}$ G. Bihlmayer, ${ }^{6}$ D. Wortmann, ${ }^{6}$ \\ G. Li ${ }^{5}$ W. Hanke, ${ }^{5}$ R. Claessen, ${ }^{1}$ and J. Schäfer ${ }^{1, *}$ \\ ${ }^{1}$ Physikalisches Institut und Röntgen Center for Complex Materials Systems, Universität Würzburg, 97074 Würzburg, Germany \\ ${ }^{2}$ Swiss Light Source, Paul-Scherrer-Institut, 5232 Villigen, Switzerland \\ ${ }^{3}$ Physik-Institut, Universität Zürich, 8057 Zürich, Switzerland \\ ${ }^{4}$ Advanced Light Source, Lawrence Berkeley National Laboratory, Berkeley, California 94720, USA \\ ${ }^{5}$ Institut für Theoretische Physik und Astronomie, Universität Würzburg, 97074 Würzburg, Germany \\ ${ }^{6}$ Peter Grünberg Institute and Institute for Advanced Simulation, Forschungszentrum Jülich, 52425 Jülich, Germany
}

(Received 4 August 2013; published 11 October 2013; corrected 5 June 2014)

\begin{abstract}
We report on the epitaxial fabrication and electronic properties of a topological phase in strained $\alpha$-Sn on InSb. The topological surface state forms in the presence of an unusual band order not based on direct spin-orbit coupling, as shown in density functional and $G W$ slab-layer calculations. Angle-resolved photoemission including spin detection probes experimentally how the topological spin-polarized state emerges from the second bulk valence band. Moreover, we demonstrate the precise control of the Fermi level by dopants.
\end{abstract}

DOI: $10.1103 /$ PhysRevLett.111.157205

PACS numbers: 75.70.Tj, 71.20.Gj, 73.20.At, 79.60.Dp

Two- and three-dimensional (2D, 3D) topological insulators (TIs) owe their conductance to spin-polarized edge or surface states, respectively, [1-4]. Assuming time-reversal symmetry, an inversion between occupied and unoccupied bands of different parity is usually caused by strong spinorbit (SO) coupling, which alters the topological invariant $\mathbb{Z}_{2}$ from $\nu=0$ (trivial insulator) to $\nu=1$ (topological insulator) [5,6]. This gives rise to an odd number of gapless boundary states [7]. For such a state, time-reversal symmetry demands that electrons moving in opposite directions carry opposite spins, which are therefore protected against backscattering. They promise lossless transport and new avenues for spin-based data processing.

A number of materials were proposed as TIs based on parity inversions [6]. Some have been investigated by angleresolved photoemission (ARPES), which can directly probe the topological surface state (TSS) with its two linearly dispersing bands that cross at the Dirac point. Examples are $\mathrm{Bi}_{0.9} \mathrm{Sb}_{0.1}$ [8], $\mathrm{Bi}_{2} \mathrm{Se}_{3}$ [9], $\mathrm{Bi}_{2} \mathrm{Te}_{3}$ [10], and $\mathrm{Sb}_{2} \mathrm{Te}_{3}$ [11] ( $\mathrm{Bi}_{2} \mathrm{X}_{3}$ family). A 2D TI is predicted [12] for $\mathrm{HgTe}$ quantum wells embedded in CdTe. Closely related to HgTe in terms of band structure is $\alpha$-Sn, which in strained form (together with $\mathrm{HgTe}$ ) has been proposed as a 3D TI [6]; however, this has not yet been explored. The band order in both high- $Z$ systems (with zero gap unless strained) is rather uncommon: the crystal symmetries together with relativistic corrections give rise to the necessary inversion of band parity-unlike in the $\mathrm{Bi}_{2} \mathrm{X}_{3}$ family where the $\mathrm{SO}$ partner bands are connected by the TSS [13].

In $\mathrm{HgTe} / \mathrm{CdTe}$ quantum wells the conductivity of $\sigma=$ $2 e^{2} / h$ is explained by spin-polarized edge channels in the quantum spin Hall effect $[3,14,15]$, giving proof of a $2 \mathrm{D}$ TI. A crossover to 3D has been reported for thick HgTe films, judged from odd Hall plateaus [16] as well as ARPES data of a linear surface state [16,17]. However, a direct probe of the spin character by spin-resolved photoemission is missing [17]. Moreover, the delicate growth together with the toxicity of $\mathrm{Hg}$ makes handling of this system difficult.

The material in focus of the present work, $\alpha$-Sn on InSb, is likewise a heteroepitaxial strained system. The use of InSb as a template for high quality crystals is well established [18-20]. It induces a slight compressive strain of $0.14 \%$ in the diamond lattice of $\alpha$-Sn. The bulk band structure of $\alpha-\mathrm{Sn} / \mathrm{InSb}$ was the subject of a few photoemission reports for the (001) [21,22] and (111) surface $[23,24]$. These early studies at low resolution, however, did not address the question of a TSS. To date, experimental and computational demonstrations are still missing.

In this Letter, we report on a 3D topological phase in strained $\alpha$-Sn. By comparing ARPES including spin detection with density functional theory (DFT) and $G W$ quasiparticle calculations, we show that the topological situation emerges from an unusual band order not simply explained by SO coupling. The spin character of the TSS is probed directly, showing a counterclockwise in-plane rotation for the occupied states of the Dirac cone. In addition, we demonstrate control of the Fermi level by adding dopants. This is the first realization of an elemental TI, which promises ease of fabrication and tuning.

$\alpha$-Sn samples have been synthesized in situ by molecular-beam epitaxy in ultrahigh vacuum on a clean $\mathrm{InSb}(001)$ substrate with an additional Te dopant flux, leading to high surface quality as monitored by low-energy electron diffraction. ARPES measurements have been performed with He-I and Ne-I excitation (21.22 and $16.85 \mathrm{eV}$ ), 


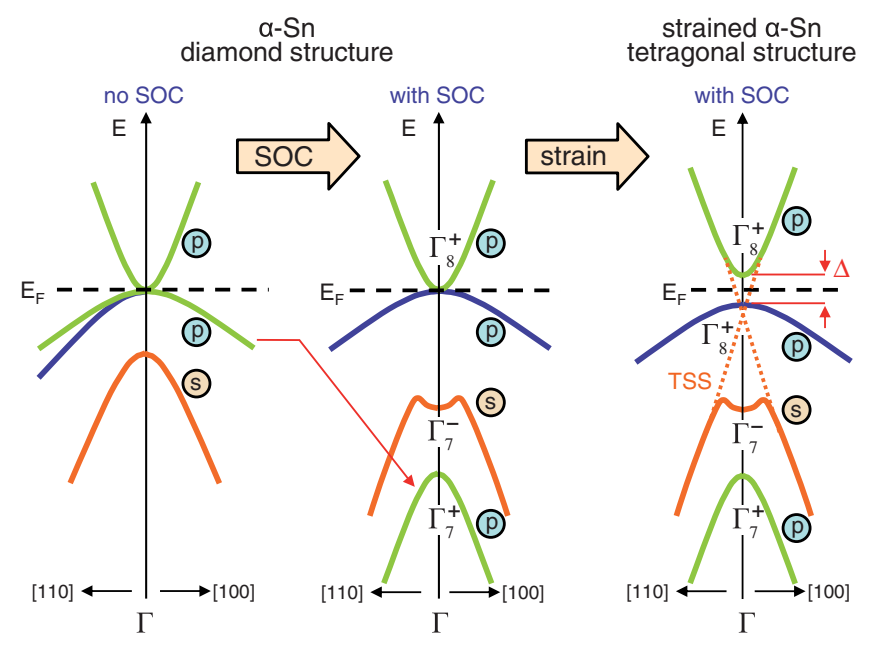

FIG. 1 (color online). Band schematics of $\alpha$-Sn for different situations without and with SO coupling (SOC), and with additional strain (double group representations refer to the diamond structure only, but are also used as band labels). Parity inversion exists independent of the presence of SOC as an inherent band feature. The TSS is hosted between the second valence band $\left(\Gamma_{7}^{-}\right)$and conduction band, while the deep SO split-off band $\left(\Gamma_{7}^{+}\right)$ is not involved. Strain opens a small band gap at the $\Gamma$ point

and by tunable synchrotron radiation. Experimental details as well as the numerical procedures are described in the Supplemental Material [25].

In $\alpha$-Sn, the band situation is different from the $\mathrm{Bi}_{2} \mathrm{X}_{3}$ TIs, where the SO coupling drives the parity inversion [13]. The unstrained $\alpha$-Sn crystal is a zero-gap material in which the light-hole valence band $\left(\Gamma_{8}^{+}\right)$and the $\Gamma_{7}^{-}$band (usually the conduction band) are inverted [26,27] compared to other semiconductors in diamond and zinc-blende structure [28] (see the band schematic in Fig. 1). The closed gap results from degenerate states at the $\Gamma$ point, where the heavy-hole valence band and the conduction band touch each other at $\Gamma_{8}^{+}$. The effect of SO coupling is to impose a strong downward shift (see the middle panel of Fig. 1), which leads to a deep-lying split-off band $\left(\Gamma_{7}^{+}\right)$.

This gives rise to the unusual band situation in $\alpha$-Sn: since the SO split-off band $\left(\Gamma_{7}^{+}\right)$is even lower than the $\Gamma_{7}^{-}$ band, this latter (second) valence band can host the TSS in conjunction with the conduction band of opposite parity. In addition, there is the first valence band $\left(\Gamma_{8}^{+}\right)$in between, representing a further modification compared to the $\mathrm{Bi}_{2} \mathrm{X}_{3}$ family with a direct (SO-driven) topological band pairing. Lattice strain then can lift the band degeneracy at the $\Gamma$ point, as shown in the right panel of Fig. 1 for in-plane compressive strain (i.e., a tetragonal lattice). A small energy gap opens at $E_{\mathrm{F}}$ while the band order remains otherwise unchanged.

The bands for bulk $\alpha$-Sn with the appropriate strain $(0.14 \%)$ have been derived from a $G W$ quasiparticle calculation (including SO coupling) and are displayed in Fig. 2(a). The strain induces a small $\Gamma$-point gap of $\sim 30 \mathrm{meV}$ at the
Fermi level. The second valence band-as the candidate to host the TSS-exhibits a weak M shape and a maximum roughly located at $0.5 \mathrm{eV}$ below $E_{\mathrm{F}}$. Interestingly, and unlike in $\mathrm{Bi}_{2} \mathrm{X}_{3}$, this carries a strong $s$-orbital character (rather than $p$ character). The third valence band (from SO coupling) is located slightly below.

In order to generate the TSS, which requires the existence of surfaces, we have performed slab-layer calculations [see Fig. 2(b)], where two linearly dispersing states (on a bulk band background) are clearly discernible [unlike simple LDA, the local density approximation plus on-site repulsion $U(\mathrm{LDA}+U)$ used here produces the correct band order, as does $G W]$. In addition, we have evaluated the spin character, which shows a clear separation into opposite signs (fully in plane for the main symmetry lines).

(a) Bulk strained $\alpha-S n(G W+S O)$

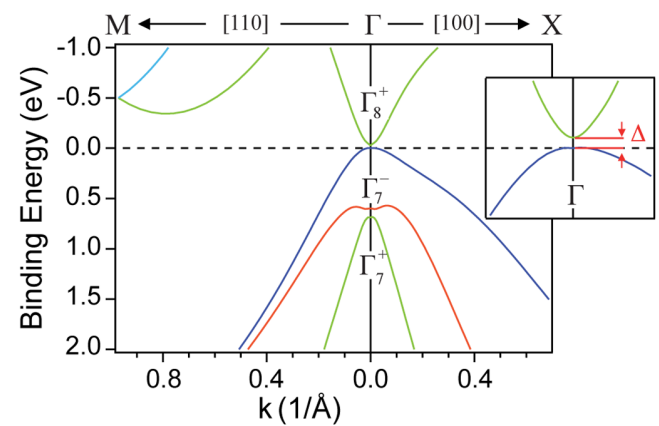

$\begin{array}{ll}\text { (b) 52-layer slab } \alpha-S n & \text { (c) Parametrized GW+SO }\end{array}$

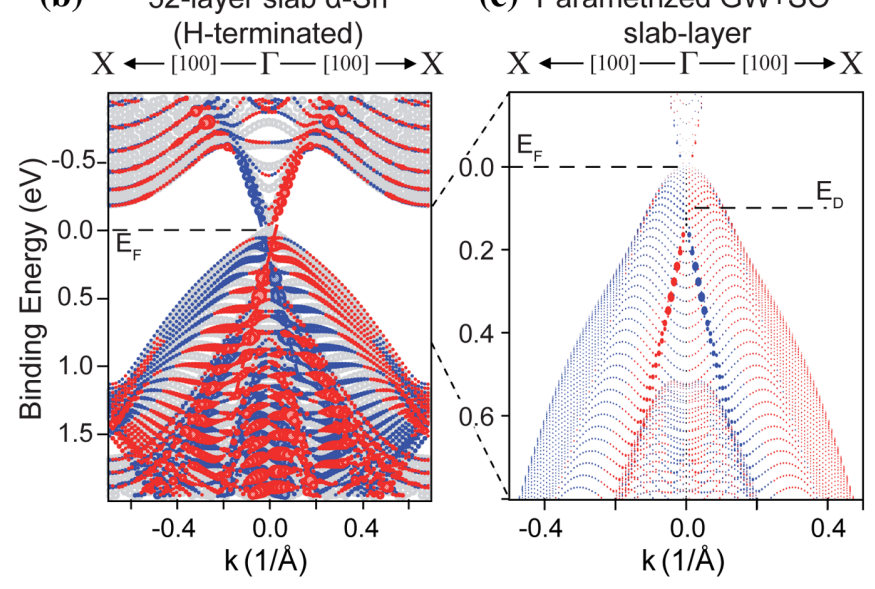

FIG. 2 (color online). (a) $G W$ quasiparticle calculation for bulk $\alpha$-Sn with realistic strain for InSb substrate $(0.14 \%$ compressive). A small gap ( $\sim 30 \mathrm{meV})$ opens at the $\Gamma$ point. (b) Slablayer LDA $+U$ calculation for the (001) surface (52 layers, H-terminated) that shows the TSS with linear dispersion. Opposite spin character is coded in red and blue, respectively, reflecting a counterclockwise spin orientation around the Dirac cone. (c) Close-up view of the TSS bands, based on a slab calculation performed in 256 layers using a tight-binding parametrization of a bulk $G W$ quasiparticle calculation (see Supplemental Material [25]). The TSS is clearly seen to emerge from the second valence band (colors: spin; symbol size: surface character within topmost 30 layers) with a Dirac point $\sim 0.1 \mathrm{eV}$ below $E_{\mathrm{F}}$. 
The spin helicity is counterclockwise below the Dirac point, and reversed above. Quantization effects present in small slab models can be avoided using a semi-infinite bulk connection to the surface layers, as in Fig. S1 in the Supplemental Material [25]. A detailed view of the TSS is obtained from $G W$ calculations where a tight-binding parametrization was implemented in a particularly thick slab with 256 layers. Here it becomes apparent that the TSS emerges straight from the second valence band, as assumed above based on the band parity sequence. It gives rise to a Dirac crossing at about $0.1 \mathrm{eV}$ below the Fermi level.

In Fig. 3(a) we present ARPES data of pristine $\alpha$-Sn taken with an excitation energy of $\mathrm{h} \nu=119 \mathrm{eV}$, which corresponds approximately to the $\Gamma$ point of the bulk BZ and, thus, compares directly to the band structure calculations of Fig. 2. We identify the $\Gamma_{8}^{+}$and $\Gamma_{7}^{-}$bands, which appear rather intense at this photon energy. Interestingly, the $\Gamma_{7}^{-}$band looses much of its intensity close to normal emission, i.e., $k=0$, which is caused by parity-related matrix elements and, thus, directly reflects the uneven parity at $\Gamma$. However, the linearly dispersing feature seen in our DFT calculations is not resolved at $119 \mathrm{eV}$ in this sample.
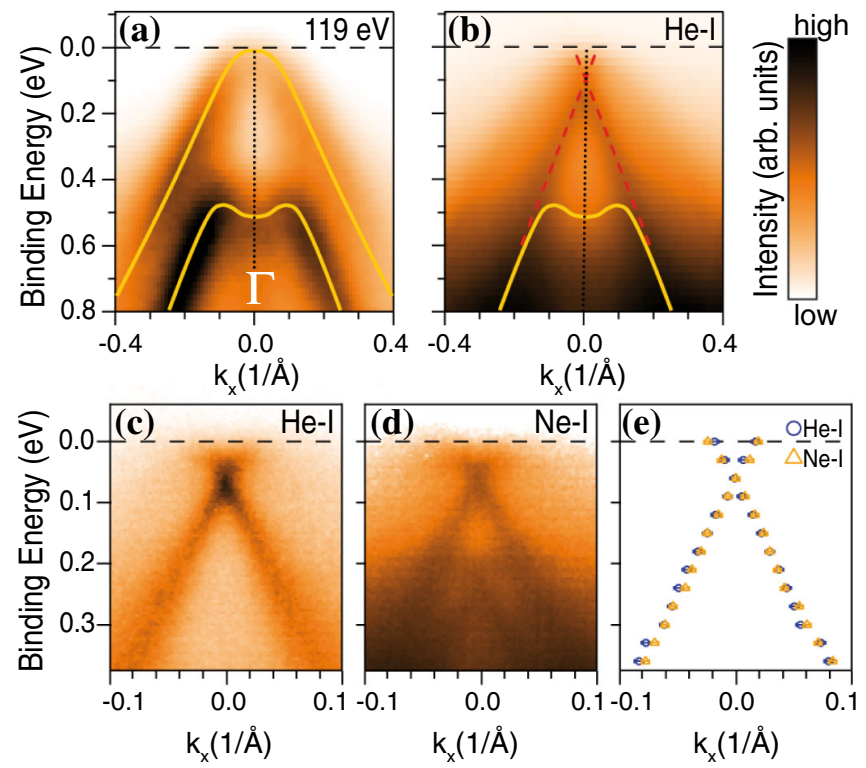

FIG. 3 (color online). (a) ARPES measurements on $\alpha$-Sn, performed at $119 \mathrm{eV}$, i.e., at the $\Gamma$ point of the $\mathrm{BZ}$, resolve the $\Gamma_{8}^{+}$and the $\Gamma_{7}^{-}$valence bands, marked by yellow lines. (b) At $\mathrm{h} \nu=21.22 \mathrm{eV}(\mathrm{He}-\mathrm{I})$ a linearly dispersing feature is observed and marked by red dashed lines. (c)-(e) Photon-energy dependent measurements, i.e., at $\mathrm{h} \nu=21.22 \mathrm{eV}$ (c) and $16.85 \mathrm{eV}$ $(\mathrm{Ne}-\mathrm{I})(\mathrm{d})$ clarify the surface character of the state. Blue circles and orange triangles in (e) represent the fitted peak positions from (c) and (d), respectively. Note that the sample of (b)-(e) is doped with Te, as described in the text. All measurements taken along the $\bar{\Gamma}-\bar{X}$-direction at room temperature in our home lab (b)-(e) and at the ALS (a). The faint replicas at higher binding energies in (d) are due to a satellite line in the Ne discharge.
The situation changes drastically at $\mathrm{h} \nu=21.22 \mathrm{eV}$ which gives a better observation condition for the soughtafter linear feature [see Fig. 3(b)]. Additionally, the sample of Fig. 3(b) has an increased surface quality as compared to the one of Fig. 3(a), since Te, which acts as a surfactant, was added during the growth, as discussed below. The change of $\mathrm{h} \nu$ adjusts the perpendicular momentum of the photoelectrons $k_{z}$ close to the $X$ point of the bulk BZ. The $\Gamma_{8}^{+}$valence band is smeared out to a weak background intensity but, centered around $\bar{\Gamma}$ of the surface BZ, we now find a prominent feature that seems to cross $E_{\mathrm{F}}$ with a rather linear dispersion. In addition, the close-up view in Fig. 3(c) suggests a crossing of the two visible branches at a binding energy of $\sim 60 \mathrm{meV}$. In agreement with our calculations presented in Fig. 2, the state emerges out of the $\Gamma_{7}^{-}$valence band, suggestion that it is indeed a TSS. We can experimentally justify this interpretation further, as we

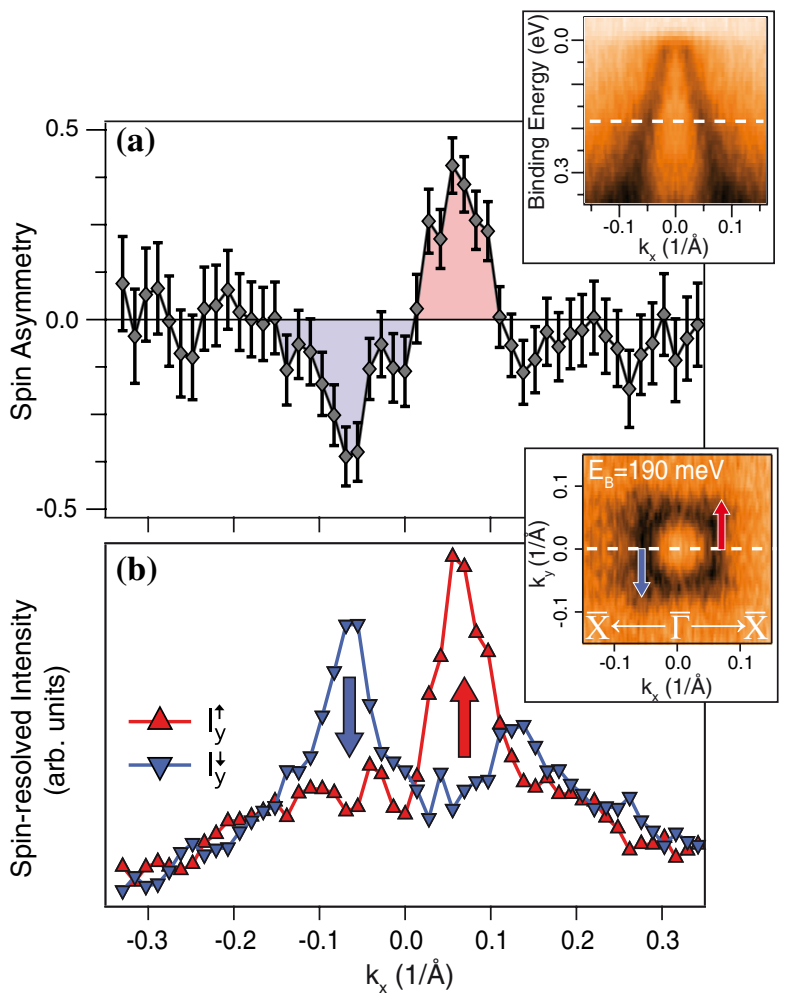

FIG. 4 (color online). Spin-resolved photoemission on $\alpha$-Sn. (a) Mott polarimetry of an MDC at $\mathrm{E}_{B}=190 \mathrm{meV}$ along the

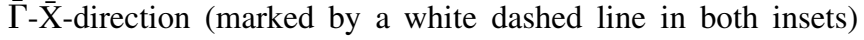
reveals a strong spin asymmetry that we assign to the surface state. (b) The derived spin-resolved intensity distribution gives one highly spin-polarized peak per spin direction, i.e., a spin down peak $(\downarrow)$ at $-k_{x}$ and a spin up peak $(\uparrow)$ at $+k_{x}$ (blue and red triangles and lines, respectively). The polarization vector points fully along the $y$ direction, which gives a counterclockwise spin helicity as shown in the constant energy surface in the lower inset. All measurements at $\mathrm{h} \nu=19.5 \mathrm{eV}$ and at $T \sim 20 \mathrm{~K}$. Spinresolved and spin-integrated measurements were performed at COPHEE and HRPES endstations at SLS, respectively. 


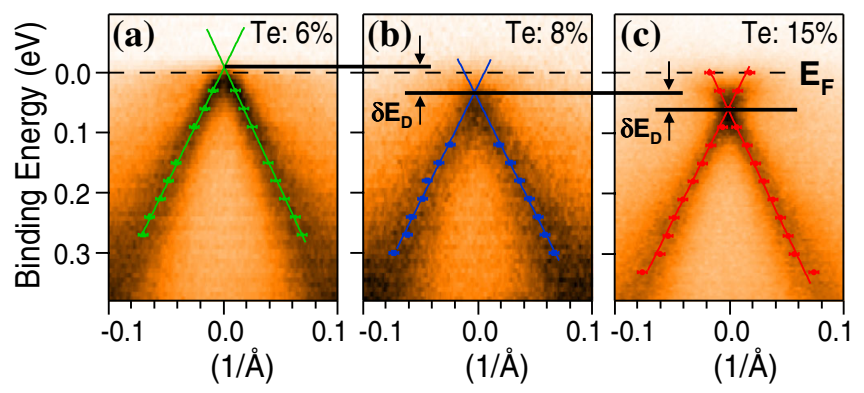

FIG. 5 (color online). Doping control. On varying the Te concentration, we shift the Dirac point from $10 \mathrm{meV}$ above $E_{\mathrm{F}}$ (a) for a Te concentration of $6 \%$ to $30 \mathrm{meV}$ (b) and $60 \mathrm{meV}$ (c) below $E_{\mathrm{F}}$ for Te concentrations of $8 \%$ and $15 \%$, respectively. Colored lines in (a)-(c) are linear regressions to the fitted MDC peak positions (colored symbols). All measurements taken along the $\bar{\Gamma}-\overline{\mathrm{X}}$ direction, at $\mathrm{h} \nu=21.22 \mathrm{eV}$, and at room temperature in our home lab.

characterize the feature to be a $2 \mathrm{D}$ surface state by changing the photon energy to $\mathrm{h} \nu=16.85 \mathrm{eV}$ [see Fig. 3(d)]. A surface state should be unaffected by this change as it shows no dispersion perpendicular to the surface. Indeed, as we quantify by an overlay of the fitted momentum distribution curve (MDC) peak positions [see Fig. 3(e)], the state is essentially unaltered by this change.

Hence, we find the linear state to satisfy key characteristics of a TSS that are surface localization and metallicity. Final evidence is obtained from the spin polarization of the surface state. For a TI the two branches of the state should show opposite spin polarization, in agreement with timereversal symmetry. In Fig. 4, we present our results from spin-resolved photoemission measurements on $\alpha$-Sn. A MDC at $E_{\mathrm{B}}=190 \mathrm{meV}$, as marked in the upper inset of Fig. 4, reveals a strong spin asymmetry when Mott polarimetry is applied to the photoelectrons [see Fig. 4(a)]. We observe two features of opposite sign, which match very well with the peak positions of the TSS in $k$ space. This leads to a spin-resolved intensity distribution where the state at $k_{x}>0$ has spin along $+k_{y}$ whereas the state at $k_{x}<0$ has spin along $-k_{y}$ [see Fig. 4(b)].

While we have measured all three spatial components of the spin vector [29], we limit our presentation to the $y$ component, as the polarization lies, within the error of the measurement, fully in this direction. Since the MDC is taken along the $k_{x}$ direction this means that the spin is oriented perpendicular to the momentum along the high-symmetry direction $\bar{\Gamma}-\bar{X}$. As sketched on the constant energy surface at $E_{\mathrm{B}}=190 \mathrm{meV}$ (see the lower inset of Fig. 4) the sign of the spin vector is such that the helicity is counterclockwise below the Dirac point, in agreement with our own DFT calculations and findings in other TIs [11,30,31].

Finally, we want to address the role of the Te adatoms during the growth of our samples. As observed by low energy electron diffraction (see Fig. S2 in the Supplemental Material [25]), the overall surface quality of our samples is increased as Te is added. This indicates that Te acts as a surfactant during the growth of $\alpha$-Sn films, similar to findings in Ge epitaxy [32]. The benefit of Te for the surface quality is further underpinned by the fact that we were not able to observe the TSS in samples without any Te added as in Fig. 3(a). We speculate that then a minor surface quality smears out the TSS in our photoemission experiment similar to observations in $\mathrm{Bi}_{2} \mathrm{Se}_{3}$ [33].

Importantly, we find that $\mathrm{Te}$ acts as an electron donor, which we demonstrate by dispersions measured on three samples with different Te concentrations [see Fig. 5]. The concentration is controlled by the Te flux during the growth and determined by core-level intensity ratios of $\mathrm{Sn}$ and Te. By increasing the Te concentration from $\sim 6 \%$ [see Fig. 5(a)] to $\sim 15 \%$ [see Fig. 5(c)], we can shift the position of the Dirac point from $\sim 10 \mathrm{meV}$ above $E_{\mathrm{F}}$ to $\sim 60 \mathrm{meV}$ below $E_{\mathrm{F}}$. We attribute the slight $p$ doping at low $\mathrm{Te}$ concentrations to a diffusion of In atoms from the substrate into the sample.

In conclusion, we have established the 3D topological insulator phase in strained $\alpha$-Sn by means of spin- and angle-resolved photoemission accompanied by $a b$ initio theoretical calculations. We find good overall agreement with relativistic LDA $+U$ as well as $G W$ quasiparticle calculations. Our results show that the band order in $\alpha$-Sn is different from the known TIs of the $\mathrm{Bi}_{2} \mathrm{X}_{3}$ family of materials. As a consequence, a surface state emerges out of the second valence band $\Gamma_{7}^{-}$that shows all characteristics of a TSS.

The close similarity of the $\alpha$-Sn bulk band structure to $\mathrm{HgTe}$ makes our system a promising candidate to exhibit the quantum spin Hall effect, if the film thickness is reduced to the $2 \mathrm{D}$ limit. In this context, it may be important to note that very recently, Sn monolayers were suggested to exhibit the quantum spin Hall insulator phase with a bulk band gap of approximately $300 \mathrm{meV}$, which would allow room temperature applications [34]. Moreover, the underlying diamond lattice of $\alpha$-Sn and the fact that it is the first realization of an elemental topological insulator opens up easier pathways of engineering future devices.

We are grateful for discussion with Oliver Rader and Laurens Molenkamp. This work was supported by the Deutsche Forschungsgemeinschaft under Grants No. FOR 1162 and No. SCHA 1510/5-1. We thank the Jülich Supercomputing Centre (JSC) for providing the computer resources. The calculations have been done at JSC within project hwb03.

*Joerg.Schaefer@physik.uni-wuerzburg.de

[1] X.-L. Qi and S.-C. Zhang, Rev. Mod. Phys. 83, 1057 (2011).

[2] C. L. Kane and E. J. Mele, Phys. Rev. Lett. 95, 226801 (2005). 
[3] M. König, S. Wiedmann, C. Bruene, A. Roth, H. Buhmann, L. W. Molenkamp, X.-L. Qi, and S.-C. Zhang, Science 318, 766 (2007).

[4] L. Fu, C. L. Kane, and E. J. Mele, Phys. Rev. Lett. 98, 106803 (2007).

[5] C. L. Kane and E. J. Mele, Phys. Rev. Lett. 95, 146802 (2005).

[6] L. Fu and C. L. Kane, Phys. Rev. B 76, 045302 (2007).

[7] C. L. Kane, Nat. Phys. 4, 348 (2008).

[8] D. Hsieh, Y. Xia, L. Wray, D. Qian, A. Pal, J.H. Dil, J. Osterwalder, F. Meier, G. Bihlmayer, C. L. Kane, Y. S. Hor, R. J. Cava, and M. Z. Hasan, Science 323, 919 (2009).

[9] Y. Xia, D. Qian, D. Hsieh, L. Wray, A. Pal, H. Lin, A. Bansil, D. Grauer, Y.S. Hor, R. J. Cava, and M. Z. Hasan, Nat. Phys. 5, 398 (2009).

[10] Y. L. Chen, J. G. Analytis, J.-H. Chu, Z. K. Liu, S.-K. Mo, X.-L. Qi, H.J. Zhang, D.H. Lu, X. Dai, Z. Fang, S. C. Zhang, I. R. Fisher, Z. Hussain, and Z.-X. Shen, Science 325, 178 (2009).

[11] C. Pauly, G. Bihlmayer, M. Liebmann, M. Grob, A. Georgi, D. Subramaniam, M. R. Scholz, J. SánchezBarriga, A. Varykhalov, S. Blügel, O. Rader, and M. Morgenstern, Phys. Rev. B 86, 235106 (2012).

[12] B. A. Bernevig, T. L. Hughes, and S.-C. Zhang, Science 314, 1757 (2006).

[13] H. Zhang, C.-X. Liu, X.-L. Qi, X. Dai, Z. Fang, and S.-C. Zhang, Nat. Phys. 5, 438 (2009).

[14] C. Brüne, A. Roth, E. G. Novik, M. König, H. Buhmann, E. M. Hankiewicz, W. Hanke, J. Sinova, and L.W. Molenkamp, Nat. Phys. 6, 448 (2010).

[15] C. Brüne, A. Roth, H. Buhmann, E. M. Hankiewicz, L. W. Molenkamp, J. Maciejko, X.-L. Qi, and S.-C. Zhang, Nat. Phys. 8, 485 (2012).

[16] C. Brüne, C. X. Liu, E. G. Novik, E. M. Hankiewicz, H. Buhmann, Y. L. Chen, X. L. Qi, Z. X. Shen, S. C. Zhang, and L. W. Molenkamp, Phys. Rev. Lett. 106, 126803 (2011).

[17] O. Crauste, Y. Ohtsubo, P. Ballet, P. A. L. Delplace, D. Carpentier, C. Bouvier, T. Meunier, A. Taleb-Ibrahimi, and L. Lévy, arXiv:1307.2008v1.

[18] R. Farrow, D. Robertson, G. Williams, A. Williams, A. Cullis, G. Jones, I. Young, and P. Dennis, J. Cryst. Growth 54, 507 (1981).
[19] B. Mason and B. R. Williams, Surf. Sci. 273, L472 (1992).

[20] E. Magnano, C. Cepek, S. Gardonio, B. Allieri, I. Baek, E. Vescovo, L. Roca, J. Avila, M. G. Betti, C. Mariani, and M. Sancrotti, J. Electron Spectrosc. Relat. Phenom. 127, 29 (2002).

[21] H. Höchst and I. Hernandez-Calderon, Surf. Sci. 126, 25 (1983).

[22] P. Fantini, M. G. Betti, C. Mariani, E. Magnano, M. Pivetta, and M. Sancrotti, Surf. Sci. 454-456, 807 (2000).

[23] I. Hernandez-Calderon and H. Höchst, Surf. Sci. 152-153, 1035 (1985).

[24] P. Fantini, S. Gardonio, P. Barbieri, U. del Pennino, C. Mariani, M. G. Betti, E. Magnano, M. Pivetta, and M. Sancrotti, Surf. Sci. 463, 174 (2000).

[25] See Supplemental Material at http://link.aps.org/ supplemental/10.1103/PhysRevLett.111.157205 for more details on theoretical and experimental methods.

[26] S. Groves and W. Paul, Phys. Rev. Lett. 11, 194 (1963).

[27] F.H. Pollak, M. Cardona, C. W. Higginbotham, F. Herman, and J. P. van Dyke, Phys. Rev. B 2, 352 (1970).

[28] W. A. Harrison, Electronic Structure and The Properties of Solids (Dover, New York, 1989).

[29] J. H. Dil, J. Phys. Condens. Matter 21, 403001 (2009).

[30] S. Souma, K. Kosaka, T. Sato, M. Komatsu, A. Takayama, T. Takahashi, M. Kriener, K. Segawa, and Y. Ando, Phys. Rev. Lett. 106, 216803 (2011).

[31] Z.-H. Pan, E. Vescovo, A. V. Fedorov, D. Gardner, Y.S. Lee, S. Chu, G. D. Gu, and T. Valla, Phys. Rev. Lett. 106, 257004 (2011).

[32] O. Sakata, P.F. Lyman, B.P. Tinkham, D. A. Walko, D. L. Marasco, T. -L. Lee, and M. J. Bedzyk, Phys. Rev. B 61, 16692 (2000).

[33] R.C. Hatch, M. Bianchi, D. Guan, S. Bao, J. Mi, B. B. Iversen, L. Nilsson, L. Hornekær, and P. Hofmann, Phys. Rev. B 83, 241303 (2011).

[34] Y. Xu, B. Yan, H.-J. Zhang, J. Wang, G. Xu, P. Tang, W. Duan, and S.-C. Zhang, Phys. Rev. Lett. 111, 136804 (2013). 